\title{
Tekiz Gebeliklerde Birinci Trimester İntrakranial Translusensi Nomogramı
}

\author{
First Trimester Intracranial Translucency Nomogram in Singleton Pregnancies
}

\author{
Fatih ŞANLIKAN ${ }^{1}$, Resul ARISOY ${ }^{1}$, Koray ÖZBAY ${ }^{1}$, Altuğ SEMİZ ${ }^{1}$
}

1. Memorial Şişli Hastanesi, Kadın Hastalıkları ve Doğum Kliniği, İstanbul, Türkiye

\section{$\ddot{O Z Z E T}$}

Amaç: 11-14. gebelik haftaları için intrakranial translusensi (IT) nomogramı oluşturulması ve IT ölçümlerinin gebelik günleri, baş popo mesafesi ve bipariyatel çap ile olan ilişkisinin değerlendirilmesi.

Gereçer ve Yöntem: Kliniğimize basvuran birinci trimester'deki 119 tekiz gebe çalışmaya dâhil edildi, bu gebelerde fetüslerin IT genişliği değerlendirilip gebelik günü, $b$ aş popo mesafesi (CRL) ve bipariyatel çap (BPD)ile olan ilişkisi araştırıldı. Yapısal veya kromozomal anomali saptanan fetüsler ve çoğul gebelikler çalışma dışı bırakıldı.

Bulgular: Çalışmamızda ortalama gebelik günü $86,6 \pm 4,9$, CRL 61,6 \pm 7,2 mm, BPD 20,7 $\pm 2,3 \mathrm{~mm}$, IT genişliği $1,7 \pm$ $0,3 \mathrm{~mm}$ olarak tespit edildi. 11-14. gebelik haftaları için gebelik günlerine göre IT nomogramı oluşturuldu. Lineer regresyon analizinde IT ile gebelik günü, CRL ve BPD arasında zayıf pozitif korelasyon saptand1 (sirasiyla $\mathrm{r}=0.30, \mathrm{r} 2=0,0899, \mathrm{r} 2$ $=0,0815$ ). IT genişliğinin 50.persentil değeri 11 hafta $0-6$ gün, 12 hafta $0-6$ gün ve 13 hafta $0-6$ gün için sırasıyla $1,5 \mathrm{~mm}, 1,7$ $\mathrm{mm}$ ve $1,8 \mathrm{~mm}$ olarak saptand 1 .

Sonuç: Birinci trimesterde nuchal tranlusensi ölçümleri yapılırken eş zamanlı olarak kolaylıkla ölçülebilen IT, santral sinir sistemi anomalilerinin saptanmasında faydalı olabilen bir ölçümdür. Çalışmamızın sonuçları literatürü destekleyecek şekilde, IT'nin ilerleyen gebelik haftalarıyla beraber lineer olarak arttığını ve gebelik günleri, CRL ve BPD ile korele olduğunu göstermiştir. Elde ettiğimiz IT nomogramı kendi popülasyonumuzun değerlendirilmesinde faydalı olabilir.

Anahtar Kelimeler: intrakranial translusensi, birinci trimester, nomogram

\section{ABSTRACT}

Objective: Our aim was to create an intracranial translucency (IT) nomogram for the 11-14 weeks of gestation and to evaluate the relationships between IT measurements and gestational days, crown rump length and biparietal diameter.

Material and Methods: 119 singleton pregnant women who applied to our clinic in the first trimester were included in the study. The IT of the fetuses were evaluated and the relationship between IT measurements and gestational day, crown rump length (CRL) and biparietal diameter (BPD) was investigated.

Results: We determined the mean values for gestational day, CRL, BPD and IT as $86.6 \pm 4.9$ days, $61.6 \pm 7.2 \mathrm{~mm}, 20.7 \pm 2.3$ $\mathrm{mm} 1.7 \pm 0.3 \mathrm{~mm}$, respectively. After then an IT nomogram was formed according to pregnancy days for the11-14gestational weeks. Linear regression analysis revealed a weak positive correlation between IT and gestational day, CRL and BPD ( $r=0.30, r 2=$ $0.0899, \mathrm{r} 2=0.0815$, respectively). The 50th percentile value of IT was determined as $1.5 \mathrm{~mm}, 1.7 \mathrm{~mm}$ and $1.8 \mathrm{~mm}$ for 11 weeks 0-6 days, 12 weeks 0-6 days and 13 weeks 0-6 days, respectively.

\section{İletişim}

Sorumlu Yazar: Fatih ŞANLIKAN

Adres: Piyalepaşa Bulvarı Memorial Şişli Hastanesi 34385 Şişli, İstanbul, Türkiye

Tel: +90 (507) 1591374

E-Posta: fatihroland@hotmail.com

Makale Geliş: 05.02.2020

Makale Kabul: 17.02.2020

DOI: http://dx.doi.org/10.16948/zktipb.685282
Conclusion: IT can be easily measured simultaneously while performing nuchal translucency measurements in the first trimester, which can be useful in detecting central nervous system anomalies. The results of our study showed that IT increased linearly with advancing gestational weeks and correlated with gestational days, CRL and BPD, in accordance with the literature. The IT nomogram we obtained can be useful in evaluating our own population.

Keywords: intracranial translucency, first trimester, nomogram

\section{GİRIŞ}

Konjenital anomaliler, intrauterin gelişim evrelerinde oluşan yapısal veya fonksiyonel anormallikler olarak tanımlanır ve prenatal dönemde, doğumda veya infant döneminde saptanabilirler. En sık görülen konjenital anomaliler kalp anomalileri ve santral sinir sistemi anomalileridir. Santral sinir sistemi anomalileri içerisinde en sık görülen nöral tüp defektleridir (NTD). NTD tüm dünyada her yıl yaklaşık 300.000 doğumda bir görülen, yaklaşık 88,000 ölüme ve 8,6 milyon yaşam boyu morbidite nedeni olan bir konjenital anomalidir [1]. En s1k görülen NTD'leri miyelomeningosel (spina bifida), meningocel, encephalocele, and anencephalidir. Mortalite ve yaşam boyu önemli morbidite nedeni olabilen spina bifidanın insidansı yaklaşı 1.4/1000 'dir [2].

Spina bifida, mid trimesterde yapilan yapısal anomali taramasında "limon" ve "muz" belirtileri ile ventrikülomegali gibi indirekt kranial belirtiler veya spinal kord açıklığının direkt ultasonografik olarak görülmesi ile genellikle tanı almaktadır. Birinci trimesterde fetüsün anatomik muayenesi yaygın olarak yapılabilir hale gelmiştir. 11-14. haftalar arasında nuchal translusensi (NT) ölçümü esnasında mid-sagital planda 4. ventrikül normalde intrakranial translusensi (IT) olarak görülebilir ve özellikle açık spina bifidanın tanısı açısından faydalı olabileceği ilk olarak Chaouri ve ark. tarafından gösterilmiştir [3-4]. Posterior beyin yapılarının açık spina bifidada caudal yer değiştirmesi sebebiyle IT'nin görülememesi veya normal sinır değerlerinin altında olması açık spina bifida hakkında bilgi verebilir. Bu da erken tanı için imkân sağlayacaktır.

Birinci trimesterde yapılan IT ölçüm değerleri gestasyonel yaş ile değişkenlik göstermektedir [3]. Bu çalışmada hastanemizde 11-14. gebelik haftaları arasinda muayene edilen fetüslerin intrakranial translusensi (IT) genişliği ölçümlerinin gebelik günü (GG), baş popo mesafesi (CRL) ve bipariyatel çap (BPD) ile ilişkisinin araştırılması ve sağlıklı fetüsler için nomogramının elde edilmesi amaçland. 


\section{GEREÇ ve YÖNTEM}

Memorial Şişli Hastanesi Kadın Hastalıkları ve Doğum Kliniğinde 2018-2019 yılları arasında; 11-14. gebelik haftaları arasında fetal anatomi muayenesi yapılan 119 tekiz fetüs çalışmaya dâhil edildi.

Fetal değerlendirmede baş-popo mesafesi (CRL), BPD, fetal kalp atım hiz1, NT, nazal kemik değerlendirmesi ve IT, Voluson E8 (General Electric, Chicago, IL, USA) kullanılarak transabdominal ultrasonografi ile yapıldı. IT ölçümü mid-sagital planda beyin sapının dorsal sınırı ile 4 . ventrükülün alt sınırı olan ekojenik koroid pleksus arasında kalan alan olarak en geniş yerinden ölçüldü (Figür 1).

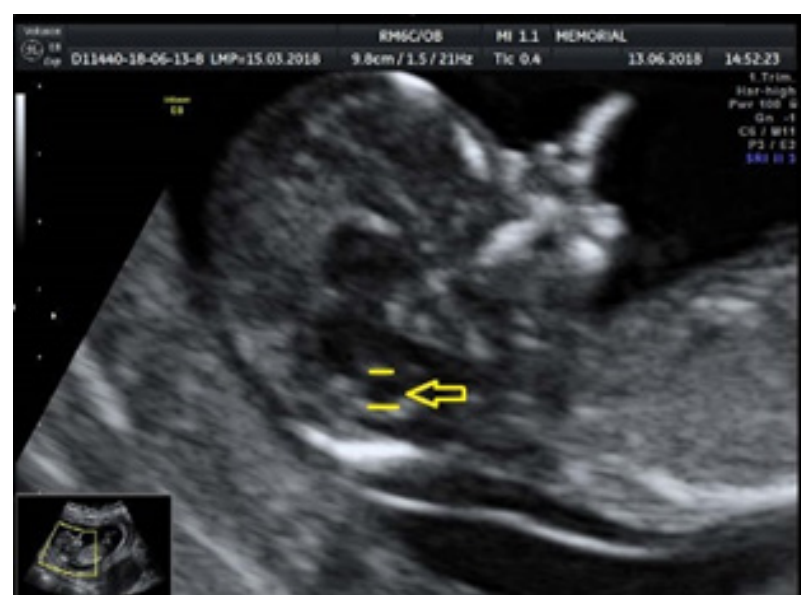

Figür 1: Mid-sagital planda IT ölçümünü gösteren ultrasonografi görüntüsü.

Yapısal veya kromozamal anomali saptanan fetüsler ve çoğul gebelikler çalışma dişı bırakıld1.

İstatistiksel analizler için SPSS programı (Version 18; SPSS Inc, Chicago, IL, USA) kullanıld. Tanımlayıcı istatiksel analizler yapıldı. IT genişliğinin gebelik günleri, CRL ve BPD ile olan ilişkileri lineer regresyon analizi ile değerlendirildi. 11-14. gebelik haftasında IT genişliği nomogramı hesaplandı. Sonuçlar \%95'lik güven aralığında, anlamlılik $\mathrm{p}<0.05$ olarak değerlendirildi.

\section{BULGULAR}

Ortalama gün $86,6 \pm 4,9$, ortalama CRL 61,6 $\pm 7,2 \mathrm{~mm}$, ortalama BPD $20,7 \pm 2,3 \mathrm{~mm}$, ortalama IT genişliği 1,7 $\pm 0,3 \mathrm{~mm}$ olarak hesapland. IT ile gebelik günü arasında zayıf pozitif korelasyon saptand1. Lineer regresyon analizinde; IT= GGx0.021-0.102 ( $\mathrm{r}=0.30 ; \mathrm{p}<0,01)$ şeklinde hesapland1 (Figür 2).

IT ile CRL ve BPD arasında da zayıf pozitif bir korelasyon saptand 1 (IT $=$ CRLx0,138+0,8264 $(\mathrm{r} 2=0,0899)$ ve $\mathrm{IT}=\mathrm{BPDx} 0,0421+0,8061(\mathrm{r} 2$ $=0,0815$ ) (Figür 3-4).

11-14. gebelik haftalarında IT genişliğinin persentil dağılımları hesaplandı. IT genişliğ̉inin 50.persentil değeri 11 hafta 0-6 gün de 1,5 mm, 12 hafta 0-6 gün 1,7 mm ve 13 hafta 0-6 gün de 1,8 mm saptand1 (Tablo 1).

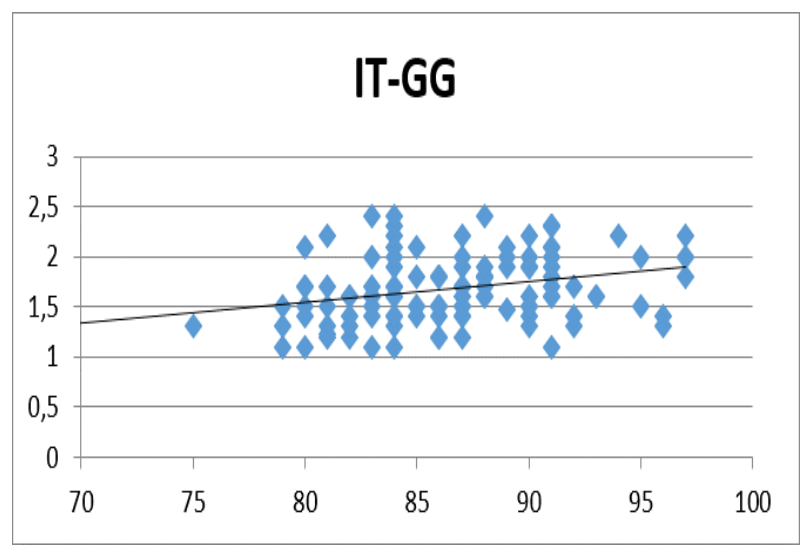

Figür 2:

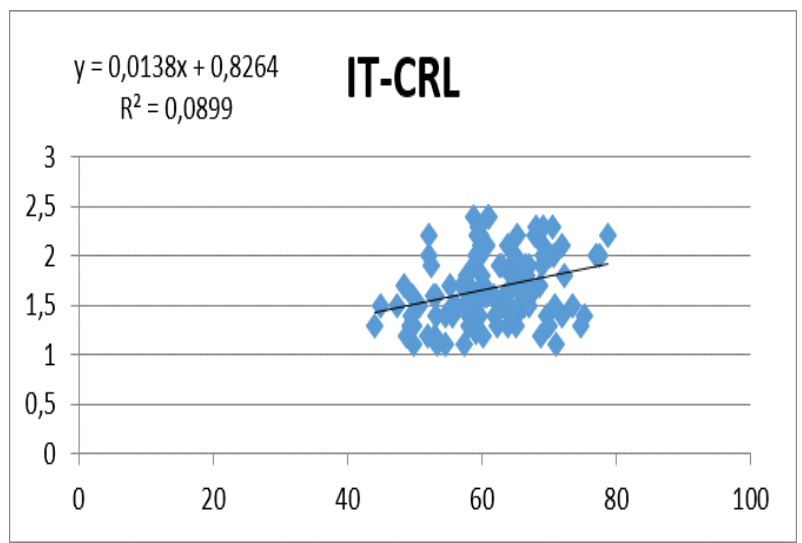

Figür 3: CRL'ye göre IT dağılım grafiği.

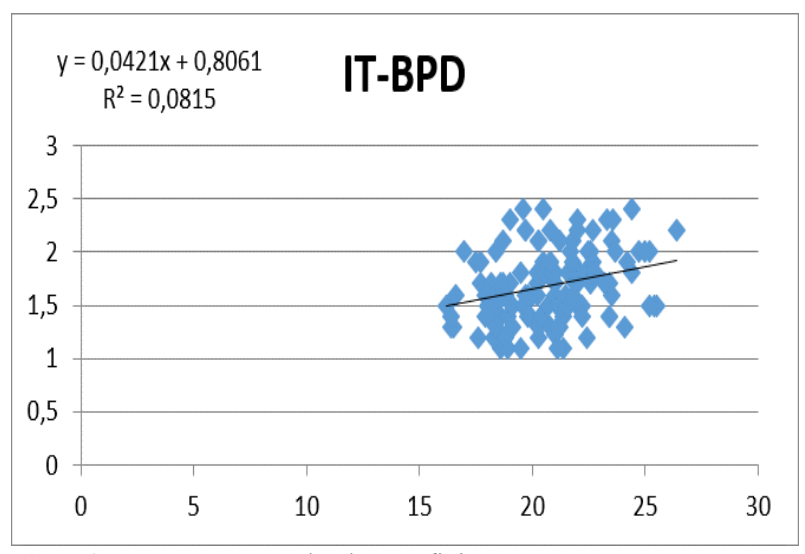

Figür 4: BPD’ye göre IT dağılım grafiği.

Tablo 1: Gebelik haftalarına göre IT persentil değerleri.

\begin{tabular}{|l|l|l|l|l|l|l|l|}
\hline \multirow{2}{*}{$\begin{array}{l}\text { Gebelik } \\
\text { Haftas } 1\end{array}$} & \multicolumn{6}{|l|}{ IT Persentil değeri } \\
\cline { 2 - 8 } & 5 & 10 & 25 & 50 & 75 & 90 & 95 \\
\hline $11^{0-6}$ & 1,1 & 1,1 & 1,3 & 1,5 & 1,7 & 2,1 & 2,3 \\
\hline $12^{0-6}$ & 1,2 & 1,3 & 1,5 & 1,7 & 1,9 & 2,2 & 2,3 \\
\hline $13^{0-6}$ & 1,2 & 1,3 & 1,6 & 1,8 & 2,1 & 2,3 & 2,3 \\
\hline
\end{tabular}

\section{TARTIŞMA}

Spina bifidanın erken tanısı için 11-14. gebelik haftaları arasında mid-sagital planda posterior beyin yapılarının incelemesi son on yılda oldukça yaygınlaşmıştır. NT ölçümü için alınan kesitte dört çizgi görüntüsü (üstten alta doğru sırasıyla beyin sapının üst sınırı, alt sınırı, 4. ventrikül koroid pleksusu ve oksipital kemik), beyin sapının posterior shifti, IT ölçümü, frontomaksiller fasial açı, sisterna magna, beyin sap1/beyin sap1-oksipital oran $(\mathrm{BS} / \mathrm{BSOB})$ ve 
biparietal çap gibi çeşitli ölçüm metotları incelenmiştir [5-11].

IT'in görülememesi açık spina bifida açısından faydalı olabileceği gibi geniş görülmesi kromozal anomali, geniş sisterna magna kisti, serebellar vermis hipoplazisi gibi anomalilerle ilişkili olabilmektedir [12-14]. IT'nin normal nomogramının belirlenmesi bu açıdan önemlidir. Türk toplumu için yapılan sınırlı sayıda çalışma vardır $[15,16]$. Bu çalışmadaki primer amacımız IT için normal sınır değerleri açısından faydalı olması amaçlanmıştır.

Kavalakis ve ark. çalışmalarında 1330 posterior beyin incelemesi yapılmış, sisterna magnanın obliterasyonu ve dört çizgi görüntüsünün normal olarak görülmemesi açık spina bifida tanısı için erken belirtiler olabileceğini fakat tüm anormal vakalarda erken beyin herniasyon bulgularının olmadığını birdirmişlerdir [5]. Çalışmalarında 1092 vakada IT ölçümü yapılmış ve ortalama $1.84 \mathrm{~mm}$ olarak bulunmuștur. Mangione ve ark. çalışmasında sisterna magnanın görüntülenemesi spina bifida için \%5073 sensivitif ve \%39-73 spesifik bulunmuş, en yüksek saptama oranının olduğunu belirtilmiştir [6]. Ferreira ve ark. trisomy 18 , trisomy 13 ve triploidi bulunan fetüslerin posterior beyin ölçümünü içeren çalışmalarında euploid fetüslerde CRL ile anlamlı olarak artan BS/BSOB oranının ciddi olarak azald1ğını göstermişlerdir [10]. Chen ve ark. 16164 fetüsü içeren çalışmalarında IT'nin komplet yokluğunda saptama oranını \%18, sisterna magnanın yokluğunda \%64 olarak belirmişlerdir [8]. Fong ve ark. 199 vakayı (191 normal fetüs ve 8 açık spina bifida) inceledikleri çalışmada 151 vakada (\%75) IT net olarak izlenmiş, IT net ya da hiç izlenmeyen 49 (\%25) fetüsün 6'sında açık spina bifidadan şüphelenilmiş, 2 normal fetüs ve 4 açık SB saptanmıştır. 43 hastada IT izlenmemesi kötü görüntülemeye bağlanmış ve bunlardan 4'ünde açık SB saptanmış. IT'nin NTD açısından sensitivitesi $\% 50$ ve spesifitesi $\% 99$ olarak bulunmuştur [7].

Literatürde IT nomogramı ile ilgili sınırlı sayıda çalıșma bulunmaktadır. Giraldo ve ark. 1520 fetüsü değerlendirilmiş, CRL ortalamasını $65 \mathrm{~mm}$ (45-84 mm), IT ortalamasını $1.7 \mathrm{~mm}$ (0.7-3.4 mm) olarak hesaplamışlardır. Çalışmamızdakine benzer olarak IT ile gestasyonel hafta arasında lineer ve pozitif bir korelasyon saptanmıștır (IT $=0,043 \times$ CRL-0,815) [17]. Adiego ve ark. 990 fetüsü içeren prospektif IT ölçümü çalıșmalarında IT ortalaması $1.8 \mathrm{~mm}$ ( $\mathrm{SD} \pm 0.37,0.8-3.1)$, gestasyonel hafta ile artan koralasyon saptamışlardır (IT=0,74+0,02xCRL; $r 2=0.15, p<0,0001)$ [18] . Aydın ve ark. çalışmalarında 2250 fetüs değerlendirilmiş, ortalama $\mathrm{CRL}=62.0 \pm 6,9$ ve $\mathrm{IT}=1,83 \pm 0,29$ olarak hesaplanmıştır [15]. Kose ve ark.'nın yaptı̆̆ı çalışmada IT ortalama 1,99 $\pm 2,6 \mathrm{~mm}$ olarak saptanmıştır.

Spina bifida saptamada IT'nin tanısal doğruluğunun değerlendirdiği meta-analize 9 çalıșma dâhil edilmiş ve toplam 21.070 fetüs'a ait verilerin analizi yapılmıştır. IT ölçümü \%97,8 (\% 95 güven aralığ 97.6-98.0) değerlendirilebilmiştir. IT'in spina bifida saptamadaki tanısal performansında duyarlılık \%53,5 ve özgüllü̈k \%99,7 olarak ölçülmüştür. Yanlış pozitiflik oranlarının yüksek olması nedeniyle spina bifida tanısı için düşük doğruluk oranlarının olmas1, ailelerde gereksiz kaygıya neden olabileceği vurgulanmıştır [20]. Yazarlar daha geniş prospektif ultrason bazlı tahmin modelleri geliştirilmesi gerektiğini vurgulamışlardır.

2019 yılında yap1lan sistematik derleme ve meta-analizde 17,598 fetüsü içeren 4 çalışmada BS/BSOB oranı ile spina bifida tanısı değerlendirilmiş ve $\mathrm{BS} / \mathrm{BSOB}$ oranının sensivitesi $0.70(95 \%$ CI $0.47-0.87$; I2=78.3\%), spesifitesi 1.00 (95\% CI $0.99-1.0 ; \mathrm{I} 2=99.2 \%)$, pozitif likelihood aran1 ve negatif likelihood oranı sirasıyla 51.44 (95\% CI 9.53$277.64 ; \mathrm{I} 2=85.5 \%)$ ve 0.23 (95\% CI $0.04-1.17$; $\mathrm{I} 2=64.8 \%)$, olarak saptanmıştır [21].

Ulhakov ve ark 2019 y1lında 1. trimester spina bifidanın yeni sonografik belirteçlerinden olabileceğini düşündükleri "crash sign” olarak tanımladıkları çalışmada retrospektif olarak tanısı doğrulanmış 53 spina bifida vakasının 48'inde mesencephalonun posteriora doğru yer değiștirmesi ve aksiyel kesitte oksipital kemiğe doğru olan deformiteyi (crash sign) doğrulamışlardır [22].

IT'nin spina bifida tanısı ile ilk yayını ortaya koyan Chaouri ve ark. 2020 y1linda choroid pleksus/ baş ölçümü oranının kullanımını gündeme getirmişlerdir. Normal fetüslerde CRL artarken koroid pleksus alanın arttığını, koroid pleksus/baş çevresinin azaldığını, tanısı doğrulanmış 38 spina bifida fetüsünde $(\% 88)$ bu oranın anlamlı derecede arttı̆̆ını saptamışlardır [23].

Birinci trimesterde NT ölçümü esnasında kolaylıkla ayırt edilebilen IT'nin değerlendirilmesi ve anormallik saptanması (IT görülmemesi veya geniş olmasi) santral sinir sistemi anomalisi hakkında uyarıcı olabilir. Çalışmamızda bulunan IT persantil değerlerinin bizim popülasyonumuzun değerlendirilmesinde katkı sağlayacağı düşünülmektedir.

\section{KAYNAKLAR}

1- Zaganjor I, Sekkarie A, Tsang BL, Williams J, Razzaghi $\mathrm{H}$ et al. Describing the Prevalence of Neural Tube Defects Worldwide: A Systematic Literature Review. PLoS One 2016;11(4):e0151586.

2- Timbolschi D, Schaefer E, Monga B, Fattori D, Dott B et al. Neural tube defects: the experience of the registry of congenital malformations of Alsace, France, 1995-2009. Fetal Diagn Ther 2015;37(1):6-17.

3- Chaoui R, Benoit B, Mitkowska-Wozniak H, Heling KS, Nicolaides KH. Assessment of intracranial translucency (IT) in the detection of spina bifida at the 11-13-week scan. Ultrasound Obstet Gynecol 2009;34(3):249-52.

4- Chaoui R, Nicolaides KH. From nuchal translucency to intracranial translucency: towards the early detection of spina bifida. Ultrasound Obstet Gynecol. 2010;35(2):133-8.

5- Kavalakis I, Souka AP, Pilalis A, Papastefanou I, Kassanos D. Assessment of the posterior brain at 11-14 weeks for the prediction of open neural tube defects. Prenat Diagn 2012;32(12):1143-6.

6- Mangione R, Dhombres F, Lelong N, Amat S, Atoub F, Friszer S, Khoshnood B, Jouannic JM. Screening for fetal spina bifida at the 11-13-week scan using three anatomical features of the posterior brain. Ultrasound Obstet Gynecol. 2013;42(4):416-20. 
7- Fong KW, Toi A, Okun N, Al-Shami E, Menezes RJ. Retrospective review of diagnostic performance of intracranial translucency in detection of open spina bifida at the 11-13-week scan. Ultrasound Obstet Gynecol 2011;38(6):630-4.

8- Chen FC, Gerhardt J, Entezami M, Chaoui R, Henrich W. Detection of Spina Bifida by First Trimester Screening - Results of the Prospective Multicenter Berlin IT-Study. Ultraschall Med 2017;38(2):151-157.

9- Suresh S, Sudarshan S, Rangaraj A, Indrani S, Cuckle H. Spina bifida screening in the first trimester using ultrasound biparietal diameter measurement adjusted for crown-rump length or abdominal circumference. Prenat Diagn 2019;39(4):314-318.

10- Ferreira AF, Syngelaki A, Smolin A, Vayna AM, Nicolaides KH. Posterior brain in fetuses with trisomy 18 , trisomy 13 and triploidy at 11 to 13 weeks' gestation. Prenat Diagn 2012;32(9):854-8.

11- Garcia-Posada R, Eixarch E, Sanz M, Puerto B, Figueras F, Borrell A. Cisterna magna width at 11-13 weeks in the detection of posterior fossa anomalies. Ultrasound Obstet Gynecol 2013;41(5):515-20.

12- Papastefanou I, Souka AP, Pilalis A, Panagopoulos P, Kassanos D. Fetal intracranial translucency and cisterna magna at 11 to 14 weeks : reference ranges and correlation with chromosomal abnormalities. Prenat Diagn 2011;31(12):1189-92.

13- Egle D, Strobl I, Weiskopf-Schwendinger V, Grubinger E, Kraxner F, Mutz-Dehbalaie IS, Strasak A, Scheier M. Appearance of the fetal posterior fossa at $11+3$ to $13+6$ gestational weeks on transabdominal ultrasound examination. Ultrasound Obstet Gynecol 2011;38(6):620-4

14- Lee MY, Won HS, Hyun MK, Lee HY, Shim JY, Lee PR, Kim A. One case of increased intracranial translucency during the first trimester associated with the Dandy-Walker variant. Prenat Diagn 2012 ;32(6):602-3

15- Sivri Aydın D, Yayla M. Evaluation of the fourth ventricle and nomogram of intracranial translucency at 11-13 weeks of gestation. Perinatal Journal 2018;26(2):102-105.

16- Ergin RN, Yayla M. The nomogram of intracranial translucency in the first trimester in singletons.

J Turk Ger Gynecol Assoc 20121;13(3):153-6.

17- Molina-Giraldo S, Pérez-Olivo JL, Arias JL, Acuña E, Alfonso D, Arreaza M, Leal MB. Normal Intracranial Translucency Values During the First Trimester of Gestation in a Latin American Population. J Ultrasound Med 2016;35(10):2231-6.

18-Adiego B, Illescas T, Martinez-Ten P, Bermejo C, Perez-Pedregosa J, Wong AE, Sepulveda W. Intracranial translucency at 11-13 weeks of gestation: prospective evaluation and reproducibility of measurements. Prenat Diagn 2012;32(3):259-63.

19- Kose S, Altunyurt S, Keskinoglu P. A prospective study on fetal posterior cranial fossa assessment for early detection of open spina bifida at 11-13 weeks. Congenit Anom (Kyoto) 2018;58(1):4-9.

20- Maruotti GM, Saccone G, D'Antonio F, Berghella V, Sarno L, Morlando M, Giudicepietro A, Martinelli P. Diagnostic accuracy of intracranial translucency in detecting spina bifida: a systematic review and meta-analysis. Prenat Diagn 2016;36(11):991-996.

21- Sirico A, Raffone A, Lanzone A, Saccone G, Travaglino A, Sarno L, Rizzo G, Zullo F, Maruotti GM. First trimester detection of fetal open spina bifida using BS/BSOB ratio. Arch Gynecol Obstet 2019 Dec 24. doi: 10.1007/s00404-019-05422-3.
22- Ushakov F, Sacco A, Andreeva E, Tudorache S, Everett T, David AL, Pandya PP. Crash sign: new first-trimester sonographic marker of spina bifida. Ultrasound Obstet Gynecol. 2019;54(6):740-745.

23-Chaoui R, Benoit B, Entezami M, Frenzel W, Heling KS, Ladendorf B, Pietzsch V, Sarut Lopez A, Karl K. Ratio of fetal choroid plexus to head size: simple sonographic marker of open spina bifida at 11-13 weeks' gestation. Ultrasound Obstet Gynecol. 2020;55(1):81-86. 